\title{
Blocking key pathway reverses brain, behavior changes in autism mouse model
}

\section{BY PETER HESS}

19 JULY 2021

Neurons in mice with an autism-linked mutation sprout extraneous protrusions, an overgrowth that tracks with above-average motor learning. The animals lose both attributes when treated with an experimental drug that suppresses the activity of the Ras-ERK/MAPK cell signaling pathway, according to a new study.

This pathway helps reshape neurons to change the strength of their connections in response to learning or other influences, part of a process known as neuroplasticity.

"There is a balance between learning and forgetting in the brain," says lead researcher Stelios Smirnakis, associate professor of neurology at Harvard University. "Understanding these pathways and how to balance them is of critical importance to a number of neurological disorders."

Hyperactivation of the Ras-ERK/MAPK pathway, which is also involved in cell growth, has been linked to cancer as well as multiple autism-related conditions.

"A lot of genes in that pathway have been shown to underlie several forms of autism," says Maria Chahrour, assistant professor of neuroscience at the University of Texas Southwestern Medical Center in Dallas, who was not involved in the study. "The pathway itself is also dysregulated in several forms of autism, so there's a potential convergence."

\section{Enhanced learning:}

The mice in the new work had an extra copy of the gene MECP2. As in previous studies and some other autism mouse models, the MECP2-duplication mice showed enhanced motor learning, mastering how to balance on a rotating rod more quickly than their wildtype counterparts. The animals' motor learning prowess offers a model for studying how the repetitive behaviors seen in people with autism develop, the researchers say. 


\section{Spectrum | Autism Research News}

https://www.spectrumnews.org

Smirnakis and his colleagues used two-photon microscopy to image neurons in the animals' motor cortex before and after four days of training on the rotating rod test.

The training prompted neurons in the MECP2 mice to grow an excess of dendritic spines - small projections on neurons that receive signals from other neurons.

Spines are usually evenly spread along dendrites. But in the MECP2 mice, they were more likely to appear in dense clusters, a sign of the animals forming stable memories, says study investigator Ryan Ash, resident in psychiatry and behavioral sciences at Stanford University in California.

The Ras-ERK/MAPK pathway is involved in the creation of spines, and was significantly more active after training in the MECP2 mice, the study also shows.

"This work adds to the increasing evidence that ERK signaling plays a probably central role in autism in general, and offers a new mechanistic explanation of the possible ways how ERK signaling is involved in the phenotype of autism," says Ning Cheng, associate professor of neuroscience at the University of Calgary in Alberta, Canada, who was not involved in the study.

The work appeared in May in eNeuro.

\section{Treatment options:}

Treating the MECP2 mice with a drug called SL327, which inhibits the Ras-ERK/MAPK pathway, restored typical motor learning and dendritic spine growth.

The drug's effects last only for about two hours, so it is unlikely to be a viable treatment for people. But some cancer drugs and cholesterol-lowering drugs called statins block the same pathway and might have therapeutic potential, Smirnakis says.

"I strongly agree with the authors that targeting ERK signaling could prove to be a fruitful approach for treating autism," Cheng says. A crucial part of this strategy will be identifying inhibitors that penetrate the blood-brain barrier and have no major side effects, she says.

Part of this search for treatments could include dissecting exactly what part of the pathway is dysregulated in individuals, leading to more personalized therapies that affect only the relevant targets, Ash says.

"This is a very essential pathway, and if you are to inhibit it, you have to be careful what other things are happening and that there are no other compensating mechanisms," Chahrour says.

SL327 did not seem to have any significant effects on spine formation in the wildtype mice, suggesting a low risk of off-target effects, Ash says. 


\section{Spectrum | Autism Research News}

https://www.spectrumnews.org

The team has constructed a library of pathways involved in neuroplasticity that might be involved in autism and other conditions, as well as in typical learning, Smirnakis says. They plan to identify which of these pathways contribute to learning in MECP2-duplication mice and test their roles in specific cell types.

Cite this article: https://doi.org/10.53053/WRGS1140 